\title{
First Measurements with the ANTARES detector
}

\section{P.Kooijman (for the ANTARES collaboration)}

University of Amsterdam, University of Utrecht, Nikhef

Science Park 105, 1098XG Amsterdam, The Netherlands

E-mail: h84@nikhef.nl

The experience of building and running a deep sea neutrino detector in the Mediterranean sea will be reviewed. The measured downward going muon flux agrees well with previously published experimental data.The first neutrino flux limits from astrophysical sources determined by the ANTARES detector will be presented. These limits are the most stringent limits to date for the sources in the southern sky, despite being determined in less than half a year data taking with a detector consisting of only five of the final twelve detector lines.

European Physical Society Europhysics Conference on High Energy Physics EPS-HEP 2009,

Krakow , Poland

July 16-22, 2009 


\section{Introduction}

Starting in February 2006 the ANTARES collaboration has steadily increased the size of its detector. This underwater neutrino telescope located at a depth of $2500 \mathrm{~m} 40 \mathrm{~km}$ south of the French city of Toulon is now comprised of its full complement of twelve detector lines. These lines are positioned at a distance of approximately $70 \mathrm{~m}$ in a configuration resembling a square surrounded by an octagon. The lines consist each of 25 storeys separated vertically by $14.5 \mathrm{~m}$, starting $100 \mathrm{~m}$ from the seafloor. Each storey houses three optical modules [1], containing a 10" photomultiplier facing downward at an angle of $45^{\circ}$ to the vertical, separated azimuthally by $60^{\circ}$. The pulseheight and timing information of all photomultiplier signals are transported to shore if the pulseheight exceeds a predetermined threshold. In most of the data presented here this was set at $30 \%$ of the mean pulseheight expected for a single photon. The lines are anchored to the seabed with a dead weight and held vertical by a buoy at the top of the line. The position and orientation of each of the optical modules is continuously monitored using a compass and tilt meters. At every fifth storey the absolute position is measured using acoustic triangulation. The detector was installed in stages with stable running periods in between submarine operations to connect several previously deployed strings. This has led to periods with 1, 2, 5, 10 and 12 lines active in the detector. The present paper describes primarily results from the period in 2007 when the detector consisted of 5 detector lines.

\section{Environmental conditions}

The environmental conditions have now been continuously monitored since February 2006. The singles count rate on the photomultipliers showed significant variation during the three years of operation. Figure 1 shows the mean counting rate in the detector as a function of time.

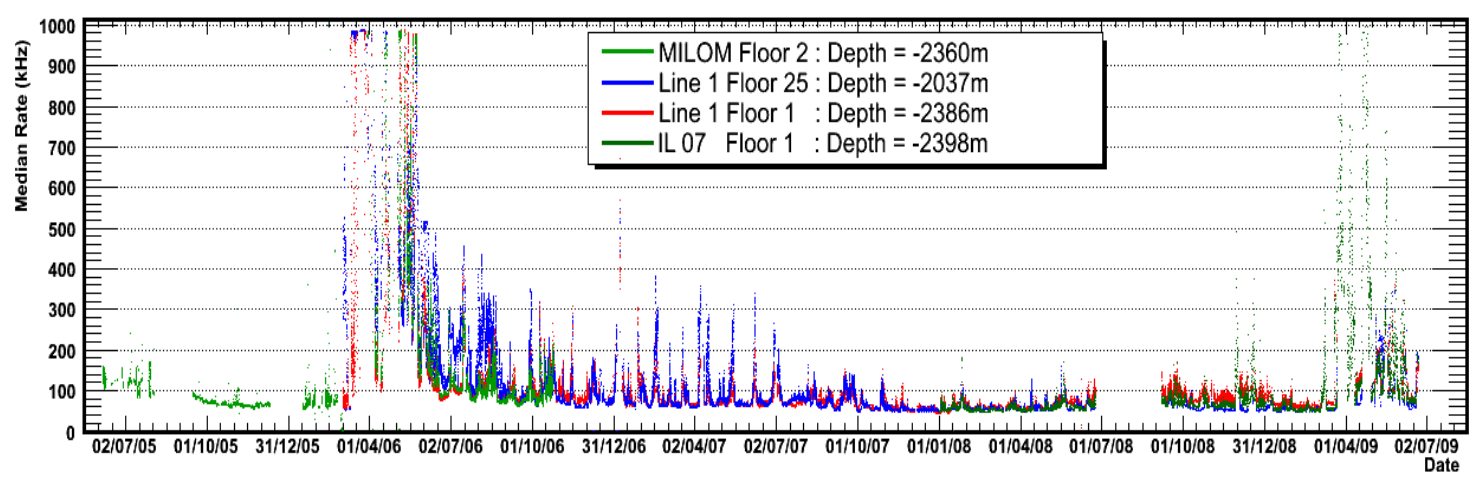

Figure 1: Singles rates as a function of time for storeys located at varying depths.

Most of the time the rate is around 50 to $60 \mathrm{kHz}$ due to the Cherenkov light produced by the radioactive decays of ${ }^{40} \mathrm{~K}$ in the seawater. There is some indication of an additional fairly 
constant rate probably due to bioluminescence. In addition to periods with this relatively constant background there are periods of extreme counting rates. These have been concentrated in two periods in the spring of 2006 and 2009. The onset of these periods coincided with extremely high water current speeds. There is keen interest in these periods from oceanographers and biologists who are also part of the ANTARES collaboration. In these periods only limited data can be taken for neutrino physics. Outside these periods, for instance form August 2006 to July 2008 and from September 2008 to April 2009, the data taking efficiency is typically above $90 \%$.

\section{Atmospheric muons}

Figure 2 shows the angular distribution of reconstructed muon tracks as a function of dip angle for 140 days of running in the five line detector configuration. The large intensity of downward going atmospheric muon tracks is evident at positive dip angles. At negative dip angles the contribution due to atmospheric neutrinos is visible. Also shown in the figure are the expectations from Monte Carlo simulations. In the figure the error on the prediction is large. At these dip angles the Cherenkov light enters the photomultiplier tubes from behind and is therefore measured on the tube at large angles with respect to the axis of the tube. At these angles the efficiency of the tube is low and not well understood. Figure 2 also shows the intensity of atmospheric muons as a function of depth as extracted from the angular distribution. It is compared to a fit to previous measurements [2] and also to the measurements performed by ANTARES with the detector consisting of only a single line [3]. There is good agreement.
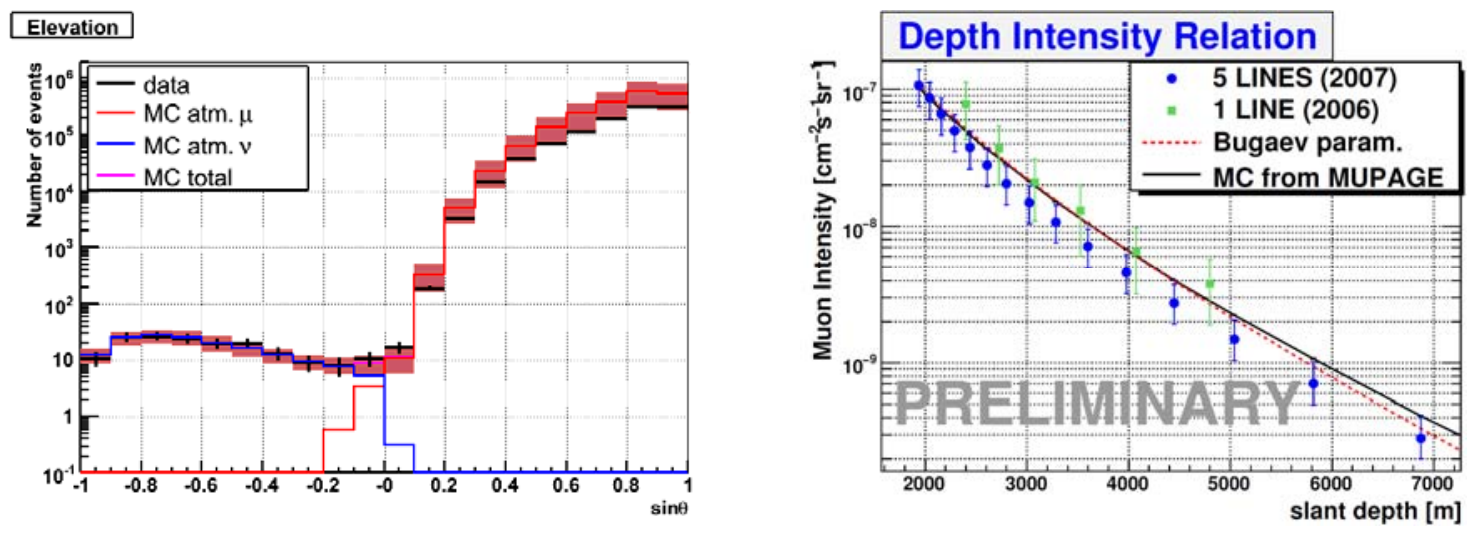

Figure 2:Angular distribution of reconstructed muons in the 5line configuration of the detector (left).The extracted intensity as a function of depth (right, 2007) compared to the Monte Carlo prediction from MUPAGE and the Bugaev parameterization. Also shown are the data extracted from the one line detector configuration (2006).

\section{Point Sources}

The upward going tracks in figure 2 which originate from neutrinos passing through the earth and interacting near the detector to produce the reconstructed muons have been used to search for signals from a selected sample of known astrophysical objects. A likelihood method 
has been used to search for a statistical excess of tracks originating from a collection of 20 sources. No excess has been observed and the absence of signal has been transformed into a limit of the neutrino flux coming from these sources. Figure 3 shows the limits obtained from the 140 days of data taking in the 5 line configuration. Also shown are the limits from MACRO [4], Super-Kamiokande [5] and the AMANDA II [6] experiments. The present limits from ANTARES are already at the level of those from other experiments that are sensitive to sources in the southern sky. Also shown is the expectation of the sensitivity for one year data taking with the detector in its full 12 line configuration.

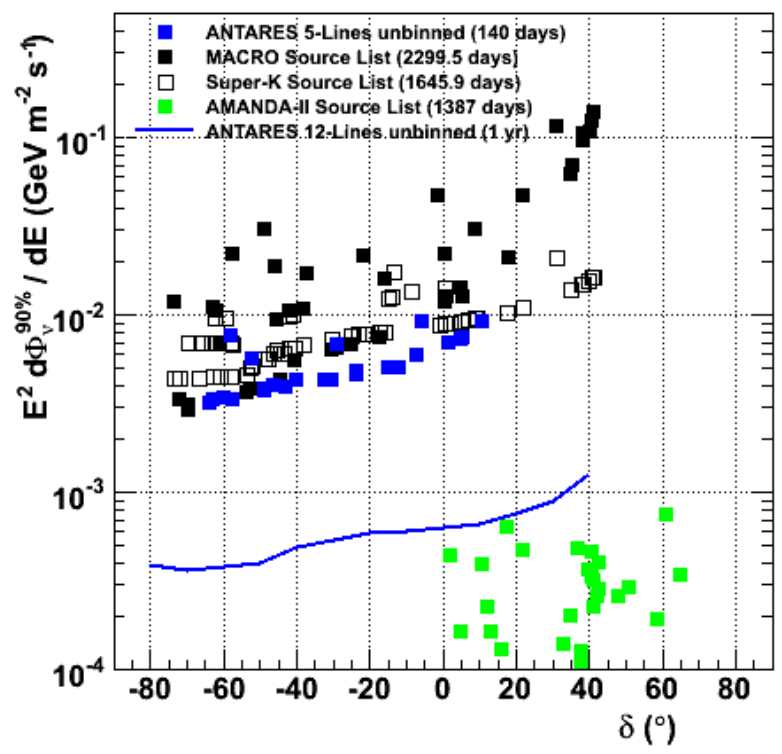

Figure 3:Flux limits for 20 selected sources. The data is for 140 days of data taking with the 5 line configuration. Also shown are the data from MACRO, Super-Kamiokande and AMANDA II.

\section{Conclusions}

The ANTARES detector is operational. We have presented data on downward going atmospheric muons which agree with the world data. The flux limits for sources are reaching or even surpassing the best limits available for sources in the southern sky.

\section{References}

[1] P.Amram et al. (ANTARES) Nucl. Instr. and Methods A484 (2002)

[2] E.V.Bugaev et al. Phys. Rev. D58, 054001

[3] M. Ageron et al. (ANTARES) Astroparticle Physics 31(4) (2009) 277

[4] M.Ambrosio et al. (MACRO) Astrophysics J. 546 (2001)1038

[5] S. Desai et al. (SuperKamiokande) Astrop. Phys. 29 (2008) 42.

[6] R.Abassi et al. (AMANDA II), Phys. Rev. D 79, 062001 (2009) 